\title{
Sportverletzungen im Vollkontakt- und Semikontaktkarate (Ein Vergleich von Kyokushinkai- und traditionellem Karate)
}

\author{
Klaus Greier, Herbert Riechelmann, Julia Ziemska
}

\section{Zusammenfassung}

Hintergrund: Karate erfreut sich sowohl im Profi- als auch im Breitensport großer Beliebtheit. Die Einteilung der verschiedenen Stilrichtungen erfolgt anhand des Impacts in Voll-, Halb- und Lowkontakt. Ziel dieser Arbeit war die Analyse von Sportverletzungen im Kyokushinkai- (Vollkontakt) und traditionellem Karate (Semikontakt).

Methoden: Im Rahmen einer Querschnittsbefragung wurden insgesamt 215 aktive Amateurkarateka (114 Vollkontakt- und 101 Semikontaktkarateka) mittels eines standardisierten Fragebogens nach sportarttypischen Verletzungen der letzten 36 Monate befragt. Die Verletzungen wurden nach Verletzungsschwere in vier Gruppen unterteilt. Schweregrad I bedeutete, dass keine ärztliche Behandlung erfolgte, Schweregrad II beinhaltete eine einmalige ärztliche Behandlung, Schweregrad III eine mehrfache ambulante medizinische Behandlung und bei Schweregrad IV erfolgte eine stationäre Behandlung.

Ergebnisse: 217 Verletzungen wurden im Einzelnen beschrieben wobei 125 Verletzungen (58\%) bei Vollkontaktund 92 Verletzungen (42\%) bei Semi- kontaktkarateka auftraten. Es ergab sich eine expositionszeitbezogene Verletzungsrate von 1,9/1000 Stunden für Vollkontakt- und 1,3/1000 Stunden für Semikontaktkarateka $(p<0,05)$. Die häufigsten Verletzungsarten waren muskuloskelettale Kontusionen (33\% Vollkontaktkarate; 20\% Semikontaktkarate), gefolgt von Distorsionen mit 19 bzw. 16\%. Die untere Extremität war bei den Vollkontaktkarateka mit $40 \%$ doppelt so häufig betroffen wie bei Semikontaktkarateka (20\%). $80 \%$ der Vollkontakt- und $77 \%$ der Semikontaktkarateka gaben an, sich während des Trainings verletzt zu haben. Sowohl im Training als auch im Wettkampf kam es beim Kumite (Freikampf) zu den meisten Verletzungen. 75\% der Verletzungen bei Vollkontaktund $70 \%$ bei Semikontaktkarateka entfielen auf leichte Verletzungen (Grad I oder II).

Schlussfolgerung: Die hohe Verletzungsrate im Training und im Kumite (Freikampf) weist auf spezifische Präventionsziele hin. Hier sollte verstärkt auf propriozeptives Training und auf ein konsequentes Aufwärmen Wert gelegt werden. Zur Prävention im Wettkampf selbst kommt den Schiedsrichtern eine wichtige Aufgabe zu.

\section{Einleitung}

Karate $($ Karate-Do $=$ der Weg der leeren Hand) hatte seinen Ursprung in China und erst zu Beginn des 20. Jahrhunderts entstand in Japan ein Kampfsport mit eigenem Regelwerk [14]. Diese Sportart

Dieser Artikel wurde erstmalig publiziert in Sportverl Sportschad 2014; 28: 31-35.

OP-JOURNAL 2016; 32: 15-19

(C) Georg Thieme Verlag KG Stuttgart · New York DOI http://dx.doi.org/10.1055/s-0042-110667

Neben dem traditionellen Karate haben sich aber auch andere Stilrichtungen entwickelt, wobei vor allem das Vollkontaktkarate zu nennen ist. Zählt Shotokan zur populärsten Stilrichtung im Semikontaktkarate, so ist Kyokushinkai die dominierende Stilrichtung im Vollkontaktkarate. Diese spezielle Kampfsportart wurde von Masutatsu Oyama in den 50er-Jahren des vergangenen Jahrhunderts in Japan aus verschiedenen Karatestilrichtungen entwickelt [6]. Bei allen Karatearten besteht das Basistraining aus drei Elementen, die man als Grundlage betrachten kann, auf die immer weiter aufgebaut wird. Die einzelnen Fundamente sind dabei die Grundschule (Kihon), der Schattenkampf (Kata), sowie der Freikampf (Kumite) (Abb. 1 und 2). Um das Karatetraining in seiner Komplexität zu perfektionieren, müssen alle drei Elemente ständig trainiert werden, wobei sich bei den Karateka häufig ein gewisser Trainingsschwerpunkt als Tendenz feststellen lässt. Als Wettkampfdisziplinen gelten Kata und Kumite [13].

Der Hauptunterschied zwischen beiden Karatestilen liegt darin, dass es im Semikontaktkarate beim Kumite (Freikampf), nie zu vollem Körperkontakt kommt, vielmehr wird kurz vor Berührung des Gegners der Schlag, Tritt oder Stoß abgestoppt, beziehungsweise nur eine leichte Berührung zugelassen (sogenannte NonContact-Regel), wo hingegen beim Kyokushinkai-Karate voller Kontakt zum Körper erlaubt ist. Zudem darf der Gegner beim traditionellen Karate nicht am Kopf getroffen werden [14]. Faustschläge zum Kopf sind wegen der hohen Verletzungsgefahr zwar auch im Vollkontaktkarate verboten, Fußtritte zum Kopf (Abb.2) sind hingegen eine häufige angewandte Technik um eine Wertung bei Wettkämpfen zu erzielen [13]. Auch bzgl. Schutzausrüstung unterscheiden sich beide Stilrichtungen. Werden im traditionellen Karate beispielsweise Zahn-, Faust-, Schienbein- und Fußschoner getragen, so sind diese im Kyoku- 


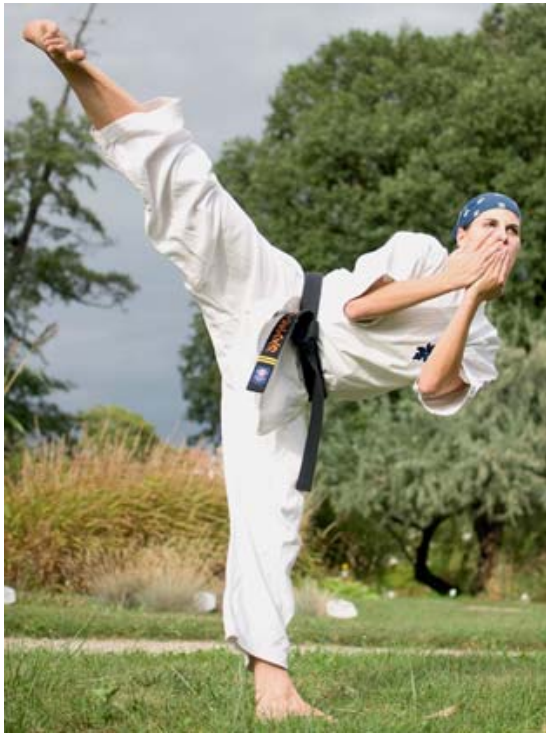

Abb. 1 Kataübung mit hohem Seitkick.

shinkai-Karate nicht vorgesehen. Männer müssen in beiden Karatestilen einen Tief- und Frauen einen Brustschutz tragen. Bei jugendlichen Karateka sind zusätzliche Schutzvorkehrungen erforderlich $[5,13]$.

Wissenschaftliche Studien $[9,17]$ identifizierten das traditionelle Karate im Vergleich mit anderen asiatischen Kampfsportarten als Sportart mit relativ geringem Verletzungsrisiko. Zetaruk et al. [17] stuften beispielsweise das Verletzungsrisiko bei Taekwondo dreimal so hoch ein. Hingegen gibt es nur sehr wenige Untersuchungen im Kyokushinkai-Karate, wodurch Daten über Entstehungsmechanismus, Art und Häufigkeit von Verletzungen bei Vollkontaktkarateka kaum vorliegen. Ziel dieser Arbeit war es deshalb, den Kenntnisstand über Verletzungsformen, -ursachen und -häufigkeiten bei Voll- und Semikontaktkarateka zu erweitern.

\section{Material und Methode}

Aktive österreichische Amateurkarateka wurden mithilfe eines standardisierten Onlinefragebogens zu sportartspezifischen Verletzungen befragt. Der Link zum Fragebogen wurde zwischen Februar und März 2013 auf der Plattform des Österreichischen Karatebundes (ÖKB) „My Dojo“ zu Verfügung gestellt. Bedingungen für eine Teilnahme waren eine mindestens dreijährige Ausübung des Sportes sowie eine Mindestaltersgrenze von 15 Jahren. Neben anthropometrischen Daten wurden individuelle Trai-

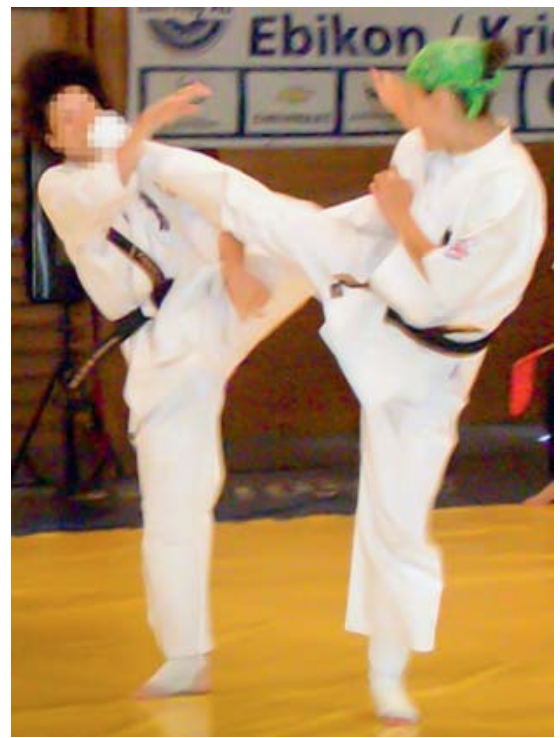

Abb. 2 Kumite im Kyokushinkai-Karate mit erlaubter Fußtechnik zum Kopf.

ningsintensität und Leistungsniveau mithilfe von durchschnittlicher Übungszeit in Stunden/Woche und Angabe des Leistungsgrades (Kyu oder Dan) ermittelt. Zudem wurden im Rahmen des Karatesports erlittenen Verletzungen der vergangenen drei Jahre (2010-2012) ermittelt. Dazu wurden Informationen zur Verletzungshäufigkeit, Verletzungsursache, Verletzungsart und Schweregrad der Verletzung eingeholt. Die Verletzungen wurden nach Verletzungsschwere in vier Gruppen unterteilt. Schweregrad I bedeutete, dass keine ärztliche Behandlung erfolgte, Schweregrad II beinhaltete eine einmalige ärztliche Behandlung, Schweregrad III eine mehrfache ambulante medizinische Behandlung und bei Schweregrad IV erfolgte eine stationäre Behandlung.

Intervallskalierte Variable wurden als Mittelwerte und Standardabweichung (SD) dargestellt. Die Anzahl der Verletzungen pro tausend Trainingsstunden wurde berechnet und mittels MannWhitney-U-Test verglichen. Korrelationsberechnungen erfolgten nach dem Pearson-Verfahren. Das alpha-Fehlerniveau wurde auf 0,05 gesetzt. Die statistischen Routinen wurden mit SPSS (Version 18) durchgeführt

\section{Ergebnisse}

Aus den 215 retournierten und vollständig ausgefüllten Fragebogen resultierte eine Datenbasis von 101 Semikontaktkarateka ( + 28\%; o $72 \%$ ) und 114 Vollkontaktkarateka ( 33\%; ơ 67\%). Das durch- schnittliche Alter, wöchentliche Trainingsstunden und Karateerfahrung in Jahren lassen sich in Tab. 1 ablesen. Fortgeschrittene Karateka mit Schwarzgurt (Dan-Träger) waren in beiden untersuchten Gruppen (Vollkontaktkarate $46 \%$; Semikontaktkarate 48\%) etwa gleich verteilt.

\section{Ursachen, Häufigkeiten, Art, Schweregrad und Lokalisation von Verletzungen}

Die Gesamtsumme von 217 beschriebenen Verletzungen verteilte sich insgesamt auf 125 Verletzungen $(57,6 \%)$ bei Vollkontaktkarateka und 92 Verletzungen $(42,4 \%)$ bei Semikontaktkarateka. Die durchschnittlich angegebene Verletzungshäufigkeit der letzten 36 Monate betrug 1,2 (Vollkontaktkarateka 1,33 vs. Semikontaktkarateka 1,06). Es ergibt sich eine expositionszeitbezogene Verletzungsrate von 1,9/1000 Stunden für Vollkontaktkarateka und 1,3/1000 Stunden für Semikontaktkarateka $(p<0,05)$. Geschlechtsspezifische Unterschiede lagen keine vor.

Ältere (31-45-jährige) Karateka erlitten weniger Verletzungen als 15-30-jährige Karateka $(r=-0,2 ; p=0,015)$.

$66 \%$ der Vollkontaktkarateka sowie 56\% der Semikontaktkarateka berichteten über mindestens eine sportartspezifische Verletzung innerhalb der letzten 36 Monate. Vollkontaktkarateka zogen sich jede fünfte Verletzung bei Wettkämpfen und $80 \%$ beim Training zu, wobei sich im Wettkampf alle Verletzungen beim Freikampf (Kumite) ereigneten. Während des Trainings kam es beim Kumite zu 56\% und durch das Schlagpolstertraining zu 14\% aller Verletzungen.

Im Semikontaktkarate ereigneten sich $23 \%$ der Verletzungen bei Wettkämpfen (95\% Kumite, 5\% Kata) und 77\% beim Training, wobei hier der Freikampf für $39 \%$ und das Kihontraining für $17 \%$ aller Verletzungen verantwortlich waren.

In der Einzelanalyse der Verletzungsarten zeigten sich muskuloskelettale Kontusionen sowohl im Vollkontaktkarate mit 33\%, als auch im Semikontaktkarate mit 20\% am Häufigsten. Distorsionen waren im Vollkontaktkarate mit 19\% und im Semikontakt mit 16\% die zweithäufigste Verletzungsart. Muskelverletzungen und Frakturen traten in beiden Karatestilen etwa gleichhäufig auf (Tab. 2). 
Die Schwere der Verletzungen, unterteilt in die Schweregrade I-IV, lässt sich aus Tab. 3 ablesen.

In Abb. $\mathbf{3}$ wird die relative anatomischtopgrafische Verletzungsverteilung von Semikontakt- und Vollkontaktkarateka dargestellt. Im Vollkontaktkarate überwiegen mit 22\% die Verletzungen der Fuß- und Zehenregion, während Handund Fingerverletzungen (14\%) im Semikontaktkarate die häufigsten Verletzungen darstellen.

\section{Diskussion}

Im Rahmen einer Onlinefragebogenerhebung wurden 215 österreichische Amateurkarateka (114 Vollkontakt (Kyokushinkai) und 101 Semikontakt) zu sportartspezifischen Verletzungen befragt. Über einen dreijährigen Zeitraum (2010-2012) kam es zu insgesamt 217 Verletzungen (125 Vollkontakt vs. 92 Semikontakt). Mehrere Studien $[9,13,17]$ konnten belegen, dass traditionelles Karate (Semikontakt) im Vergleich $\mathrm{zu}$ anderen asiatischen Kampfsportarten ein relativ geringes Verletzungsrisiko aufweist. Das Risiko, Verletzungen zu erleiden, wird bei Taekwondo beispielsweise dreimal so hoch eingestuft [17].

Die durchschnittlich angegebene Verletzungshäufigkeit der letzten 36 Monate lag im Durchschnitt bei 1,2 Verletzungen mit einer expositionszeitbezogenen Verletzungsrate von 1,9/1000 Stunden für Vollkontakt- und 1,5/1000 Stunden für Semikontaktkarateka. Sowohl im Vollkontakt- als auch im Semikontaktkarate ereigneten sich die meisten Verletzungen im Training und beim Kumite (Freikampf). Dies kann auf die Tatsache zurückgeführt werden, dass das Verletzungsrisiko beim Freikampf mit Gegnerkontakt gegenüber den isolierten Kataübungen höher ist. Bei der Kataausübung sind die Übergänge der Techniken und Stände im Kyokushinkai-Karate eher fließend, im traditionellen Karate jedoch schneller und abrupter, was hier die höhere Verletzungsrate bei Semikontaktkataturnieren erklären könnte.

Beim Semikontaktkarate wurde auch die Grundschule (Kihon) als verletzungsprovozierendes Trainingselement angegeben, während beim Vollkontaktkarate das Schlagpolstertraining ein erhöhtes Verletzungsrisiko aufweist. Der Grund dafür könnte wohl in den unterschiedlichen Trainingsformen $\mathrm{zu}$ finden sein. Beim Vollkontaktkarate muss der Athlet

Tab. 1 Durchschnittsalter von 114 Vollkontaktkarateka und 101 Semikontaktkarateka, sowie durchschnittliche wöchentliche Trainingszeit und Karateerfahrung in Jahren.

\begin{tabular}{lcc} 
Merkmale & $\begin{array}{l}\text { Vollkontaktkarateka } \\
\text { MW (SD) }\end{array}$ & $\begin{array}{l}\text { Semikontaktkarateka } \\
\text { MW (SD) }\end{array}$ \\
\hline Alter & $31,2(S D \pm 10,6)$ & $31,6(S D \pm 12,8)$ \\
\hline Trainingsstunden/Woche & $4,5(S D \pm 2,5)$ & $5,2(S D \pm 2,7)$ \\
\hline Karateerfahrung in Jahren & $12,6(S D \pm 8,9)$ & $12,5(S D \pm 9,1)$
\end{tabular}

Tab. 2 Absolute und relative Häufigkeiten der Verletzungsart von 114 Vollkontakt- und 101 Semikontaktkarateka bei 217 Verletzungen. ${ }^{1}$

\begin{tabular}{lcc} 
Verletzungsart & Vollkontaktkarate $\mathbf{n}(\%)$ & Semikontaktkarate $\mathbf{n}(\%)$ \\
\hline 1. Frakturen & $16(13)$ & $15(16)$ \\
\hline 2. Band-/Sehnenrupturen & $8(6)$ & $7(8)$ \\
\hline 3. Meniskusverletzungen & $1(1)$ & $6(7)$ \\
\hline 4. Distorsionen & $24(19)$ & $15(16)$ \\
\hline 5. Kontusionen & $41(33)$ & $18(20)$ \\
\hline 6. Muskelverletzungen & $17(14)$ & $12(13)$ \\
\hline 7. Riss./Quetschverletzungen & $9(7)$ & $4(4)$ \\
\hline 8. sonstige & $9(7)$ & $15(16)$ \\
\hline Verletzungen insgesamt & $125(100)$ & $92(100)$
\end{tabular}

Prozentwerte wurden gerundet.

Tab. 3 Absolute und relative Häufigkeiten der Schweregrade der Verletzungen von 114 Vollkontakt- und 101 Semikontaktkarate. ${ }^{1}$

\begin{tabular}{lcl} 
Verletzungsschweregrad & Vollkontaktkarate $\mathbf{n}(\%)$ & Semikontaktkarate $\mathbf{n}(\%)$ \\
\hline Schweregrad I & $51(41)$ & $33(36)$ \\
\hline Schweregrad II & $43(34)$ & $31(34)$ \\
\hline Schweregrad III & $24(19)$ & $20(21)$ \\
\hline Schweregrad IV & $7(6)$ & $8(9)$ \\
\hline Verletzungen insgesamt & $125(100)$ & $92(100)$ \\
1 Prozentwerte wurden gerundet. & &
\end{tabular}

in der Lage sein, seinen Gegner während des Kampfes kraftvoll und ohne Scheu mit Schlägen und Tritten anzugreifen. Dies wird beispielsweise durch gezieltes Schlagpolstertraining geübt. Da dieses im Semikontaktkarate gänzlich wegfällt, hat diese Art von Training hier wenig Relevanz.

Ältere Karateka erlitten bei der vorliegenden Untersuchung weniger Verletzungen als Jüngere, dieser Zusammenhang war jedoch sehr schwach ausgeprägt $(r=-0,2)$. Die Ergebnisse verschiedener Studien sind diesbezüglich jedoch nicht konsistent. So konnte in einigen Studien $[4,16]$ beobachtet werden, dass junge und unerfahrene Kämpfer ein höheres Verletzungsrisiko aufweisen als Amateure und Professionelle. Andere Studien $[8,13,17]$ ergaben hingegen, dass ältere Kämpfer über 20 Jahren und mit mehr als drei Jahren Karateerfahrung häufiger von Verletzungen betroffen waren. Zudem stieg die Verletzungsgefahr bei einem Trainingspensum von mehr als drei Stunden pro Woche [17].

Die vorliegende Untersuchung ergab bei beiden Karatestilen bzgl. der anatomisch-topografischen Verletzungsverteilung ein ähnliches Bild (Abb. 3). Es überwiegen sowohl beim Vollkontakt-, als auch Semikontaktkarate Verletzungen der Fuß- und Zehenregion, sowie der Hand und Fingerbereiche. Die untere 


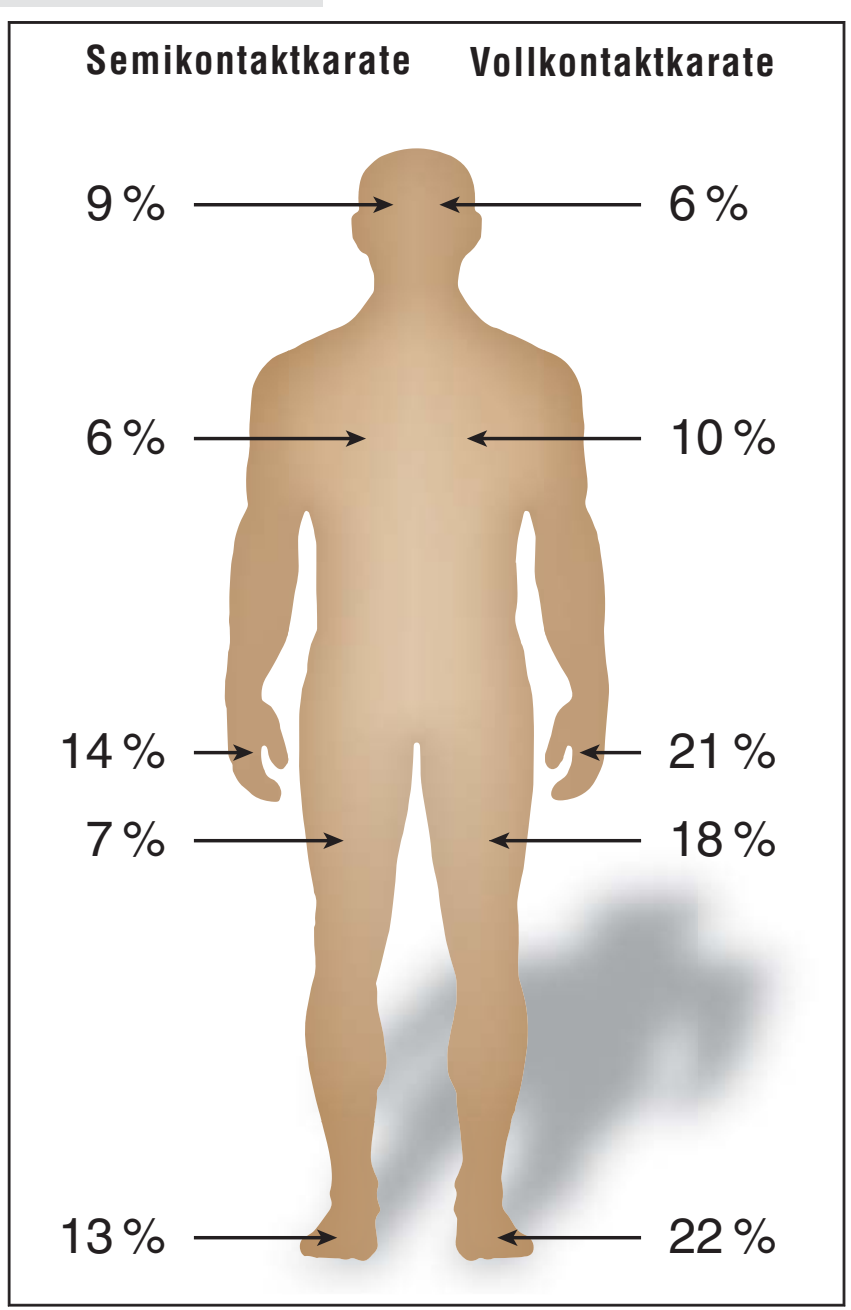

Abb. 3 Relative anatomische-topografische Verteilung von Verletzungen beim Semikontaktakt(links) und Vollkontaktkarate (rechts).

Extremität ist jedoch beim Vollkontaktkarate doppelt so häufig betroffen wie beim Semikontaktkarate.

Beim Vollkontaktkarate treten Oberschenkelverletzungen mit $18 \%$ fast dreimal so häufig auf wie beim Semikontaktkarate $(7 \%)$. Ursache dürfte der für das Vollkontaktkarate typische und effiziente Low-Kick gegen den Oberschenkel sein.

Einige Studien $[1,8,11]$ lokalisierten beim traditionellen Karate den HalsKopf-Bereich als dominierende Verletzungsregion. Dies konnte in unserer Untersuchung jedoch nicht beobachtet werden. Müller-Rath et al. [8] nannten als möglichen Grund für die häufigen Verletzungen im Kopfbereich die mangelnde Kontrolle der Karateka, die Schlagtechnik im letzten Moment abzustoppen, um den Gegner nicht, beziehungsweise nur sehr leicht, zu berühren. Die Verwendung von Faust- und Fußschützern führte beim Kumite kaum zu einer Verringerung der Verletzungsrate im Kopfbereich $[8,17]$, hingegen nahm nach Meinung von Rosso et al. [13] seit
Einführung der Schienbein- und Fußschoner die Inzidenz an schweren Verletzungen $\mathrm{ab}$.

Kontusionen stellen in der vorliegenden Untersuchung sowohl im Vollkontaktals auch im Semikontaktkarate die dominierende Verletzungsart dar, gefolgt von Distorsionen. Selbst bei Verwendung von Schutzausrüstung kann es zu Kontusionen kommen, da die Trefferfläche des Gegners sehr groß ist und diese unmöglich zur Gänze geschützt werden kann. Im traditionellen Karate sind etwa fünf bis zehn Prozent der Kampfsportler auch von chronischen Überlastungsschäden betroffen [12]. Hier wird ebenso der Fuß als häufige Verletzungsregion genannt. Die Überlastungsschäden kommen hauptsächlich von den immer wieder repetitiven Technikübungen im Kihon.

Internationale Studien $[12,13]$ belegen, dass schwerwiegende Verletzungen im traditionellen Karate einen geringen Anteil innerhalb des Verletzungsspektrums ausmachen. Harsch et al. [5] konnten in ihrer Studie nachweisen (durchgeführt während der Kyokushinkai Karate Europameisterschaft 1997), dass schwere Verletzungen auch bei Vollkontaktkarate eher selten sind.

Dies konnte in der vorliegenden Untersuchung ebenfalls beobachtet werden. Anhand anamnestischer Angaben wurden die Verletzungen nach vier Schweregraden klassifiziert. Geringgradige und leichte Verletzungen (Schweregrad I und II) dominierten sowohl im Vollkontakt- $(75 \%)$ als auch im Semikontaktkarate $(70 \%)$. Schwere Verletzungen (Grad IV) waren in beiden Karategruppen gering. Das mag auf den ersten Blick verwundern, assoziiert man doch mit Vollkontaktkarate meist eine harte und verletzungsträchtige Sportart. Dass dem nicht so sein muss, wurde häufig mit der Tatsache begründet, dass sich meist sehr erfahrene und gut ausgebildete Kämpfer gegenüberstehen. Diese weisen einen hohen konditionellen Level auf und verfügen über perfekte Abwehrtechniken [5].

\section{Präventive Maßnahmen}

Ausgedehnte Regeländerungen konnten die Verletzungsquote im Karate insgesamt nur gering beeinflussen. Es kam jedoch zu Veränderungen bzgl. Lokalisation, Art und Ursache der Verletzungen [15]. Die Verwendung eines Tiefschutzes bei Männern und eines Brustschutzes bei Frauen führte zu einer Verletzungsreduktion [14], wo hingegen die Verletzungsprävention durch Implementierung von Hand-, Schienbein- und Fußschonern weiterhin kontrovers diskutiert wird $[8,13]$.

Propriozeptives Training (z.B. Balancedisc) und nicht sportartspezifisches Training wie etwa Krafttraining, verbessern nicht nur die intramuskuläre Koordination, sondern kräftigen auch Gelenke, Rumpf- und Rückenmuskulatur, wodurch Verletzungen reduziert werden könnten [7]. Bei Wettkämpfen liegt es vor allem an den Schiedsrichtern, Verletzungen der Kämpfer zu vermeiden, indem sie Simulationen von Wirkungstreffern unterscheiden und richtige Wertungen abgeben [1].

\section{Schlussfolgerungen}

Zusammenfassend zeigt die vorliegende Untersuchung, dass vor allem schwere Verletzungen im Vollkontakt- und Semikontaktkarate selten sind. Dies wird 
auch in anderen Studien bestätigt. Um vor allem die Verletzungshäufigkeit im Training und Freikampf (Kumite) zu verringern, wird entsprechend der Ergebnisse dieser Studie empfohlen, ein umfassendes Aufwärmen, sowie ein konsequentes propriozeptives Training durchzuführen. Das vollständige Ausheilen von Krankheiten bzw. Verletzungen ist unabdingbar. Wie auch in anderen Sportarten spielt dabei das stufenweise Aufbautraining eine wichtige Rolle.

\section{Interessenkonflikt: Nein}

\section{Literatur}

${ }^{1}$ Ariazza $R$, Leyes $M$. Injury profile in competitive karate: prospective analysis of three consecutive World Karate Championship. Knee Surg Sports Traumatol Arthrosc 2005; 13: 603-607

2 Baumann P. Einblicke in die Kunst Karate-Do. Norderstedt: Books on Demand GmbH; 2012

${ }^{3}$ Chaabène H, Younés H, Franchini E et al. Physical and Physiological Profile of Elite Karate Athletes. Sports Med 2012; 42: 829-843

${ }^{4}$ Götz U, Hörterer H. Verletzungen bei Kindern und Jugendlichen im Karatesport. Sportortho Sporttraum 1999; 15: 180-181
${ }^{5}$ Harsch E, Harsch V, Stanislawski H. Kyokushinkai-Karate am Beispiel der Europameisterschaften 1997 in Berlin. Dtsch Z Sportmed 1998; 49: 124-126

${ }^{6}$ Höller J, Maluschka A. Vollkontakt-KarateTraining. Aachen: Meyer \& Meyer; 2010

7 Hubscher M, Zech A, Pfeifer Ket al. Neuromuscular training for sports injury prevention: a systematic review. Med Sci Sports Ex 2010; 42: 413-421

${ }^{8}$ Müller-Rath R, Miltner O, Mamarvar $M$ et al. Das Verletzungsrisiko im Jugend- und Juniorenwettkampfkarate. Sportverl Sportschad 2005; 19: 191-194

${ }^{9}$ Ngai K, Levy F, Hsu E. Injury trends in sanctioned mixed martial arts competition: a five-year review 2002-2007. Br J Sports Med 2008; 42: 686-689

10 Österreichischer Karatebund (ÖKB). Im Internet: www.karate-austria.at; Stand: 8.7.2013

11 Pieter W. Martial arts and injuries. Med Sport Sci 2005; 48: 59-73

12 Rosso C, Zenhäuser R, Valderrabano V. Verletzungen bei Karate-ähnlichen Kampfsportarten. Schweiz Z Sportmed Sporttraum 2010; 58: 56-57

${ }^{13}$ Rosso C, Zenhäuser R, Müller A et al. Karate-Do - The Path of the Empty Hand. Sportortho Sporttraum 2012; 28: 12-16

14 Schmitt H. Karate. In: Engelhardt M, Hrsg. Sportverletzungen. 2. Aufl. München: Urban \& Fischer; 2009: 489-491

15 Wanke E. Verletzungsprävention im Wettkampfkarate am Beispiel von Änderungen im Regelwerk. Z Angew Trainingswissenschaft 2008; 15: 142-150
${ }^{16}$ Zetaruk M, Violán $M$, Zurakowski $D$ et al. Karate injuries in children and adolescents. Accid Anal Prev 2000; 32: 421-425

17 Zetaruk M, Violán M, Zurakowski D et al. Injuries in martial arts: a comparison of five styles. Br J Sports Med 2005; 39: 29-33

\section{Institutsangaben}

Klaus Greier ${ }^{1,2}$, Herbert Riechelmann ${ }^{3}$, Julia Ziemska ${ }^{4}$

1 Pädagogische Hochschule Stams, Bewegungs- und Sporterziehung, Stams, Austria

2 Institut für Sportwissenschaft der Universität Innsbruck, Austria

3 Medizinische Universität Innsbruck, Universitätsklinik für HNO, Innsbruck, Austria

4 Fachhochschule Kufstein, Sport-, Kultur- und Eventmanagement, Kufstein, Austria

\section{Prof. PD Dr. Klaus Greier}

Leiter des Studiengangs Bewegungsund Sporterziehung

Bewegungs- und Sporterziehung Kirchliche Pädagogische Hochschule Stams

Stiftshof 1

6422 Stams

Austria

nikolaus.greier@kph-es.at 